\title{
Running head: CHANGING FACES: DIRECTION IS IMPORTANT
}

\author{
Changing Faces: Direction is Important \\ David A. Ross \\ University of Cardiff \\ Peter J.B. Hancock \\ University of Stirling \\ Michael B. Lewis \\ University of Cardiff
}

Address for correspondence:

Rossd1@cardiff.ac.uk

David Ross

School of Psychology

Tower Building

Cardiff University

Park Place

Cardiff, Wales, UK

CF10 3AT

Phone: 02920874007

Fax: 02920874854858

Author postprint, to appear in Visual Cognition: DOI: 10.1080/13506280802536656

\section{Acknowledgements}

This research was supported by grant PTA-031-2006-00064 from the ESRC. The authors would like to thank Sarah Stevenage and two anonymous reviewers for their helpful feedback and also Rob Honey, Peter Hills and John Ross for their comments on the paper.

\footnotetext{
Abstract

Recent research has provided evidence for the role of norm-based coding in face recognition (e.g. Leopold et al., 2001). In such a model, any given face can be represented by a vector from the norm, with the difference between two faces being the difference between the two vectors. However, as has previously been suggested it is also possible to conceive of a model in which the angle between two vectors provides relevant information for differentiating faces. Two experiments investigated this possibility using a face matching paradigm. Discrimination was found to be better when two faces lay on different vectors than when they were the same physical distance apart but lying on the same vector. The results favour a specific type of norm-based model suggesting that vector angle is important in face perception.
} 


\section{Changing Faces: Direction is Important}

Valentine’s (1991) 'face space’ metaphor provides a parsimonious description of distinctiveness effects in face recognition and categorisation. Faces are represented as coordinates within a multidimensional space and are generally assumed to be normally distributed along each of the dimensions. The distinctiveness of a face is therefore a function of its distance from the centre of the space with distinctive examples occupying a less densely populated region of the space than typical ones. As Valentine noted, there are two distinct ways of representing face space. First, the norm-based coding model proposes that faces are encoded as deviations from the central tendency along at least one of the face space dimensions. Second, the exemplar-based model suggests that faces are encoded only relative to other faces within the space meaning the norm or average face is purely theoretical and plays no role in the encoding process.

Determining between norm- and exemplar-based models has become a major focus of the face recognition literature. Initially exemplar-based models seemed to provide a more parsimonious explanation for a number of emerging findings. For example, Valentine and Endo (1992) demonstrated that the effect of race and distinctiveness on recognition memory was best explained by an exemplar-based model. Indeed, norm-based accounts would have to postulate that own- and other-race faces were encoded relative to distinct norms. However, recently a number of studies using adaptation aftereffects have provided further support for norm-based coding (e.g. Leopold, O’Toole, Vetter and Blanz, 2001; Robbins, McKone \& Edwards, 2007).

Leopold et al. (2001) investigated the effect that prolonged presentation of a particular face (leading to adaptation) has on the recognition of a target face. In their experiment the adaptation face was generated such that it was in the opposite direction of deviation from an average face to the deviation of the target face. In line with the predictions of a norm-based model, this opposite adaptation moved the participants' thresholds for identification closer to the average or norm: Participants could recognise the target face even when some of its identity had been reduced by moving it towards an average face. Indeed they demonstrated that the average face was now recognised $60 \%$ of the time as the target identity, as opposed to a chance level of $25 \%$ (there were four possible target faces). Furthermore, Rhodes and Jeffery (2006) have demonstrated that this adaptation aftereffect is greater following adaptation to a face that is directly opposite the target identity than when adapting to a face that is rated as equally dissimilar to the target face but which is not directly on the opposite side of the norm. These results suggest that identity is encoded relative to an average face.

\section{The Role of Vector-Angle in a Norm-Based Model}

Within a norm-based model of face space, each face is represented by its deviation from a norm face on a number of dimensions. Similarity between two faces will be dependent on the similarity of these deviations. Some formulations of the norm-based face space model will, in fact, be mathematically identical to an exemplar-based model. If similarity between two faces is wholly determined by vector similarity using vector subtraction then the difference between two faces will not depend upon the relative positive of the average or norm face (see Craw, 1995).

There is, however, another way in which we could specify the position of a face, and hence similarity of faces, within a norm based model. Rather than simply represent it as set of 
coordinates, we can also give some weighting to its direction within the face space. Indeed, this is implied by models of 'holistic processing' or indeed any model in which separate configural components or even gross features are processed in such a way that the sum of the parts is different from the whole (see Tanaka \& Farah, 2003). For example, consider the relationship between the norm face and the three faces A, B and C shown in Figure 1. Although faces $B$ and $C$ lie an equal distance from Face A they may not be equally perceptually different from Face A. This is because Face B, as a consequence of being an extension of the vector between the norm and Face A, maintains the 2:1 ratio for its value on dimension 1 to the value on dimension 2. Conversely, the ratio of value on dimension 1 to the value on dimension 2 for Face $C$ is 1:3. It follows that vector direction from the norm to a face may encode psychologically relevant information about identity such that an angle $\theta$ between two norm-face vectors will be directly related to their dissimilarity above and beyond the vector subtraction measure of dissimilarity. Another way that this situation may be phrased is that a face having a large bulbous nose might effect the perception of the eyes, perhaps making them appear smaller or closer together.

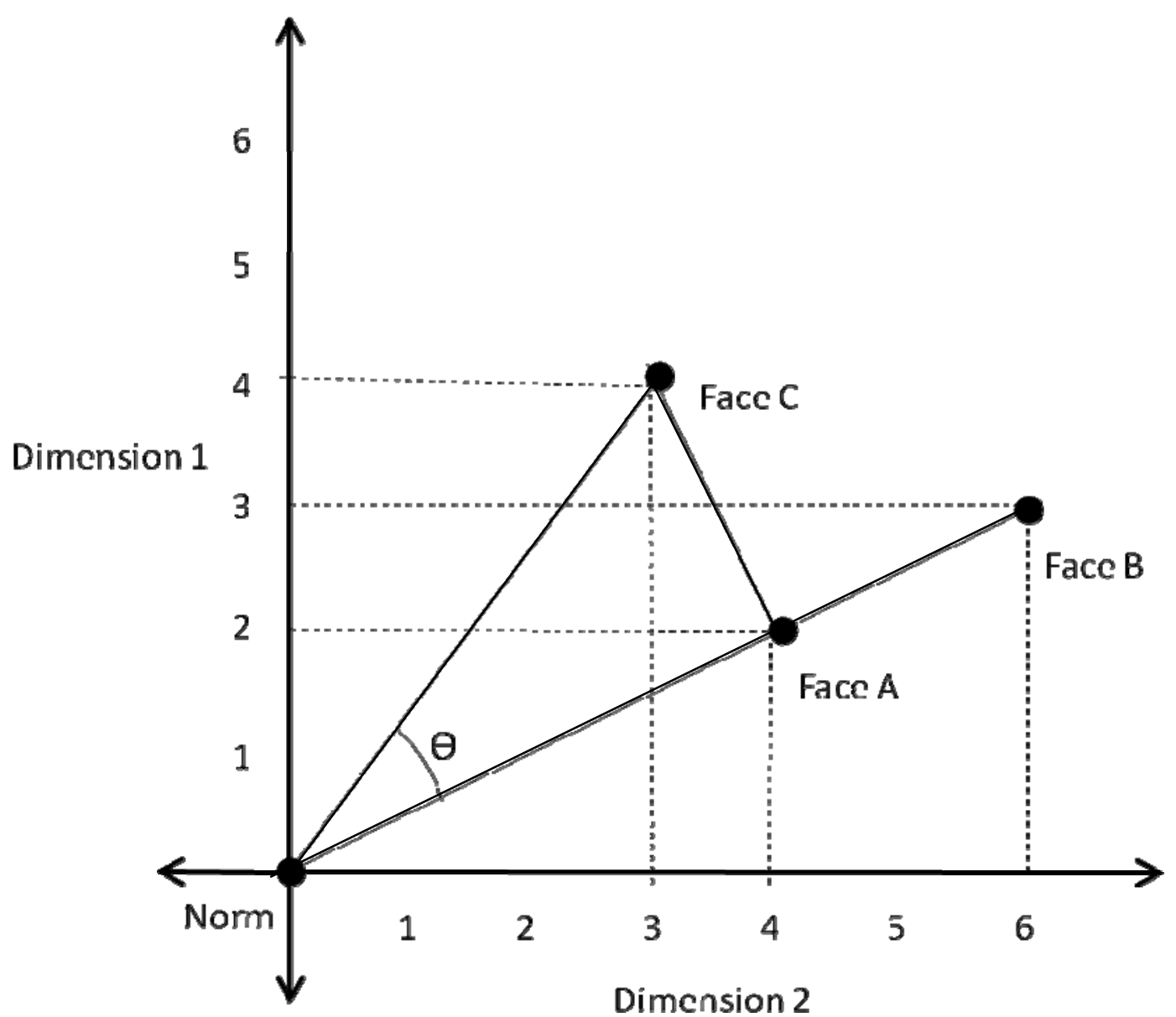

Figure 1. Three faces, Face A, Face B and Face C. Faces B and C are separated from face A by the same distance but there is an angle $\theta$ between the vector from the norm to Face $\mathrm{A}$ and the norm to face $\mathrm{C}$.

\section{Lateral Caricatures}


This idea of there being a role for the direction of deviation in facial similarity is not new. It has been implicit or explicit in almost all of the discussions of norm-based face space. One study that explicitly set out to investigate the predictions of such a norm-based model was reported by Carey (1992). Carey reported data relating to the recognisability of caricatures, anti caricatures and lateral caricatures. A caricature is created by moving a face away from the norm point in face space while maintaining the original vector direction. For example, in Figure 1, Face B is a caricature of face A. Conversely to produce an anti caricature the image is moved closer to the average face. The Lateral caricature is created by moving the image in a direction orthogonal to the vector from the norm such as can be seen for Face $\mathrm{C}$ in Figure 1. The effects of caricature and anti caricature on the recognition of known faces have been well established (see Rhodes, 1996 for a review). Caricatures are recognised more quickly than both veridicals and the equivalent anti caricatures (e.g. Rhodes, Brennan \& Carey, 1987). There is also some evidence that a slight degree of caricaturing may create an image that is recognised more accurately than the veridical, particularly if the images are degraded (Benson \& Perrett, 1994).

Carey suggested that if one was to create a caricature, an anti caricature and a lateral caricature of a known face so that each of the three new faces were equidistant from the veridical then a norm-based model would predict that the lateral caricature would be the hardest to recognise as the original person. This prediction was based on the notion that caricaturing would increase what was distinctive about the face in relation to the norm, anti caricaturing would decrease it but lateral caricaturing would change it (Carey, 1992; Rhodes, Carey, Byatt \& Proffitt, 1998). However, as was discussed there is no reason why a normbased model should necessarily predict anything other than similarity being a simple subtraction of the two norm-face vectors. That is to say that simple vector algebra would predict that faces B and C in Figure 1 are equally similar to face A. Indeed, although Carey initially reported that laterals were recognised worse than both caricatures and anti caricatures more rigorous testing has revealed that it is anti caricatures that are recognised the worst followed by Laterals and then caricatures (Lewis \& Johnston, 1998, 1999; Rhodes et al. 1998).

Although these results would seem to rule out a model in which angle of deviation has a role in differentiating two identity vectors, there may also be other reasons for the failure to find the anticipated pattern of results. First, a drawback of Rhodes et al.'s (1998) methodology is that it relied on recognition rather than perceptual similarity (this is also true for Lewis \& Johnston's, 1998, study). Although norm-based models predict that laterals will be perceptually less like the veridical than caricatures this would not necessarily translate directly into a recognition disadvantage. This is because the number of competing faces encoded in memory is greater near the centre of face space and therefore in both norm- and exemplar-based models the activation of the target will depend to some extent on how much the probe face activates other exemplars. Therefore the only way in which this technique could differentiate the two models is if the extra perceptual distance between the lateral and the target outweighed the recognition advantage provided by the caricature being in a less densely populated area of face space.

A second criticism is that the method used by Rhodes et al. to create the Lateral caricatures may have resulted in making faces that were not within the psychological face space. Indeed, it has been pointed out that the lateral caricatures produced did have a rather contorted appearance (Stevenage, 1997). This is because the laterals, like the caricatures, were created by representing points on a target identity as coordinates deviating from an average on a $2 \mathrm{D}$ plane. In a caricature each point is moved by multiplying its $\mathrm{x}$ and $\mathrm{y}$ 
coordinate by a given factor thus moving the points away from the average. Importantly when generating a caricature one can be relatively confident that the resultant face is not too unusual so long as the multiplication factor is relatively small (say 1.5). However, when creating a Lateral caricature each point can be moved one of two ways, that is to say orthogonally to the right or left of the norm-deviation vector. When Rhodes et al. created their lateral caricatures they chose to move the points on one half of the face to the right and those on the other half to the left this resulted in faces that had one eye moved up and the other down, or one eye heavily slanted and the other changed in shape. Importantly, we know that the asymmetries produced by this method are not typical in a face and indeed faces manipulated in this way may quickly leave the psychological face space (e.g. McKone, Aitkin \& Edwards, 2005; Robbins, McKone \& Edwards, 2007). On first appearances this may seem to suggest that the Lateral caricatures should have been recognised more slowly as they are less face like, however this is not necessarily the case because they were also tending towards an area of lower exemplar density. It may, at first, seem that because any two equidistant lateral caricatures created from a single veridical face will lie an equal distance from the centre of face space they should be in regions of equal exemplar density. This assumption is clearly not true however as some laterals maintain facial symmetry whereas others do not. Given the lower density of exemplars in some lateral directions one could consider some lateral caricatures could produce similar effects to caricatures therefore speeding up their recognition.

In the experiment presented here, we set out to explicitly test whether the direction of a face space vector is important for identity, that is to say does the angle between two norm face vectors play a role in determining their similarity? Rather than investigate the effect of the difference in vector angles on recognition memory, we investigated its effect on the just noticeable difference to a given face. It was hoped that by testing the perceptual space rather than the memory space we would provide a stronger test than that provided in previous studies. Indeed, a failure to find an effect of vector direction would rule out any role of angle in the determination of perceptual facial similarity.

\section{Experiment 1}

In experiment 1 we tested the size of change required to discriminate two very similar versions of unfamiliar faces. These changes were either in the direction of caricature (i.e., the angle relative to the norm was kept the same) or the change was of an equivalent physical distance in a direction that changed the angle of deviation of the face from the norm. The size of the change required to notice the difference in the images was used as a measure of similarity in that direction. The method used to generate the stimuli used in this experiment was designed to create realistic stimuli in which the effects of physical change were controlled so that the contribution of vector angle in distinguishing between two faces could be assessed.

Method

Participants. The participants were a sample of 22 undergraduates from Cardiff University, School of Psychology who signed up for the experiment as a partial fulfilment of course requirements. Participants were aged between 18 and 22 years, and all reported that they had normal or corrected to normal vision.

Materials. The face stimuli were 22 full frontal, grey-scale photographs of unfamiliar male faces selected from the Stirling database. All images were cropped to remove the background and resized to a width of 350 pixels using Photoshop ${ }^{\mathrm{TM}}$. The images were 
converted to greyscale, cropped to remove all background and resized to a width of 350 pixels in Photoshop ${ }^{\mathrm{TM}}$.

To create the experimental stimuli, the faces were first separated into 11 arbitrary pairings using the Excel ${ }^{\mathrm{TM}}$ random function. Second, an average was constructed from 100 images of male faces using Psychomorph (Tiddeman, Burt, \& Perrett, 2001). Lastly, both faces in each of the pairs were separately morphed to create two types of experimental stimuli, Caricatures and Obliques.

Caricature stimuli were constructed along the vector direction between the average face and the target faces. Figure 2 shows an example using two hypothetical faces, face $\mathrm{A}$ and face B. First, a 50\% anti-caricature of the faces in each pair was constructed, $\mathrm{A}_{\mathrm{C} 1}$ and $\mathrm{B}_{\mathrm{C} 1}$. Psychomorph was used to move 250 hand-marked data points on each face directly towards the equivalent data points marked on the average face. Similarly, two 50\% caricatures, $\mathrm{A}_{\mathrm{C} 2}$ and $\mathrm{B}_{\mathrm{C} 2}$, were created by moving each of the points on the target face further from those on the average. Two continua of 51 faces were then produced for each pair, with faces spaced in equal increments between the $50 \%$ anti-caricature and the $50 \%$ caricature, that is to say $A_{C 1}$ to $A_{C 2}$ and $B_{C 1}$ to $B_{C 2}$. As can be seen from Figure 2 the faces $A_{C 1}$ to $A_{C 2}$ lie along a single vector from the average face as do the faces $\mathrm{B}_{\mathrm{C} 1}$ to $\mathrm{B}_{\mathrm{C} 2}$. These two sets therefore lay on the caricature continuum.

The Oblique stimuli for each celebrity pair were constructed so as to pass through the same midpoint as the Caricature continuum, face $\mathrm{A}$ and face $\mathrm{B}$, but on an oblique vector. First, the data points on the two $50 \%$ anti-caricatures from each pair, $\mathrm{A}_{\mathrm{C} 1}$ and $\mathrm{B}_{\mathrm{C} 1}$, were moved away from each other by $100 \%$ to create two new faces, $\mathrm{A}_{\mathrm{O} 1}$ and $\mathrm{B}_{\mathrm{O} 1}$. The two new faces were then used to move the veridical faces, face $A$ and face $B, 100 \%$ in the opposite direction creating the last pair of faces, $\mathrm{A}_{\mathrm{O} 2}$ and $\mathrm{B}_{\mathrm{O} 2}$. The Oblique continuum was then constructed connecting the two new pairs of points, $\mathrm{A}_{\mathrm{O} 1}-\mathrm{A}_{\mathrm{O} 2}$ and $\mathrm{B}_{\mathrm{O} 1}-\mathrm{B}_{\mathrm{O} 2}$. As with the Caricature continua the Oblique continua were divided into 51 equal increments with the veridical images, face $\mathrm{A}$ and face $\mathrm{B}$ as their midpoints.

It is worth pointing out that moving the two faces $100 \%$ away from each other has no particular psychological relevance as the direction is arbitrary, based only on the relative positions of the two faces. Differences between the two faces will be exaggerated, for example if one face has a big nose and the other a smaller nose then this difference will be more extreme in the newly formed face. However, unlike when moving through the average it is possible that the distinctiveness of a feature would be unchanged but that the feature might change. For example a round nose may become pointier with neither being more distinctive than the other. 


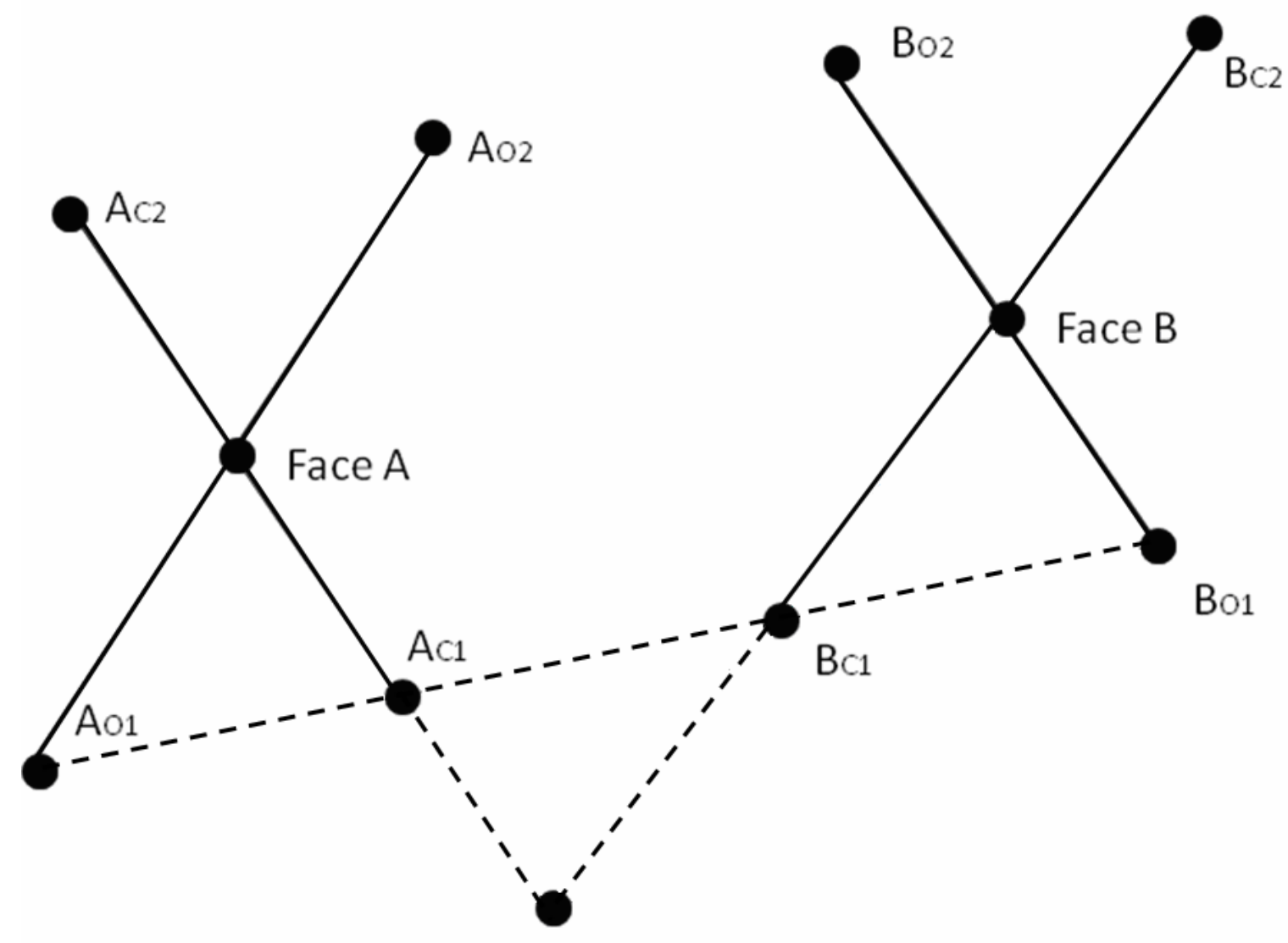

\section{Average Face}

Figure 2. The two Caricature continua $\mathrm{B}_{\mathrm{C} 1}-\mathrm{B}_{\mathrm{C} 2}$ and $\mathrm{A}_{\mathrm{C1}}-\mathrm{A}_{\mathrm{C} 2}$ and the two Oblique continua $\mathrm{A}_{\mathrm{O} 1}-\mathrm{A}_{\mathrm{O} 2}$ and $\mathrm{B}_{\mathrm{O} 1}-\mathrm{B}_{\mathrm{O} 2}$. Note that continuum $\mathrm{A}_{\mathrm{O} 1}-\mathrm{A}_{\mathrm{O} 2}$ is the same length as and parallel to the continuum $\mathrm{B}_{\mathrm{C} 1}-\mathrm{B}_{\mathrm{C} 2}$ and continuum $\mathrm{A}_{\mathrm{C} 1}-\mathrm{A}_{\mathrm{C} 2}$ is the same length as and parallel to $\mathrm{B}_{\mathrm{O} 1}-\mathrm{B}_{\mathrm{O} 2}$. In this way, equivalence of physical changes within pairs of stimuli was guaranteed. One participant would be tested on continua $\mathrm{A}_{\mathrm{O} 1}-\mathrm{A}_{\mathrm{O} 2}$ and $\mathrm{B}_{\mathrm{C} 1}-\mathrm{B}_{\mathrm{C} 2}$ where as a second would be tested on continua $\mathrm{A}_{\mathrm{C} 1}-\mathrm{A}_{\mathrm{C} 2}$ and $\mathrm{B}_{\mathrm{O} 1}-\mathrm{B}_{\mathrm{O} 2}$.

The actual physical distance between the two end points of the Caricature continuum for face $\mathrm{A}$ is the same as the distance between the two end points of the Oblique continuum for face B (as represented in Figure 2). Essentially this is because two parallelograms have been created with the two parallel vectors forming the opposite sides. The 11 pairs of faces , each of which contained two oblique continua and two caricature continua, were then split into 22 sets such that each set contained 51 faces along a caricature continuum and 51 faces from the corresponding oblique continuum, that is to say, the oblique continuum of the same length. As an illustration of the effect, suppose one face has a rather large nose. Caricaturing will cause the nose to become even larger. This same change will be applied in the Oblique continuum to the second face, which may have a normal nose. The second face is thus altered in exactly those dimensions in which the first face is distinctive (see Figure 3 for an example of the stimuli). 
3a)
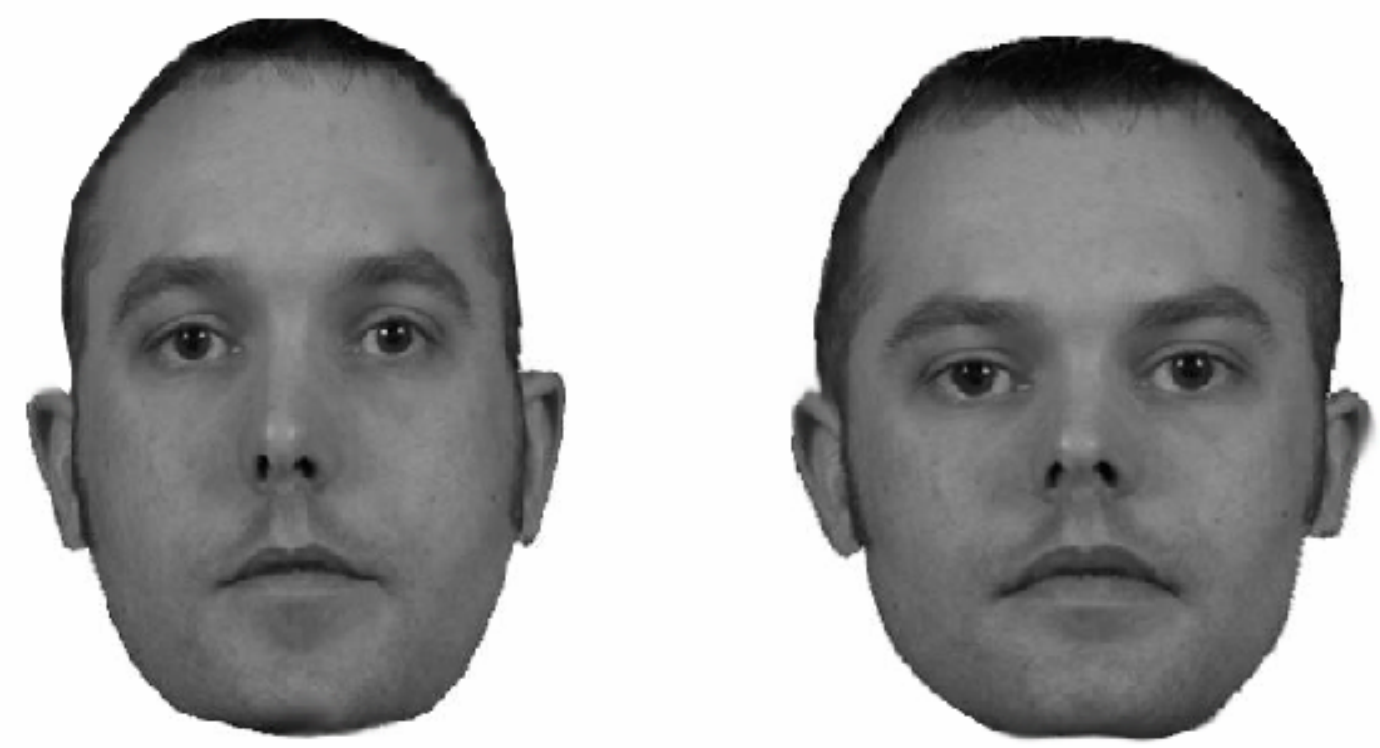

3b)
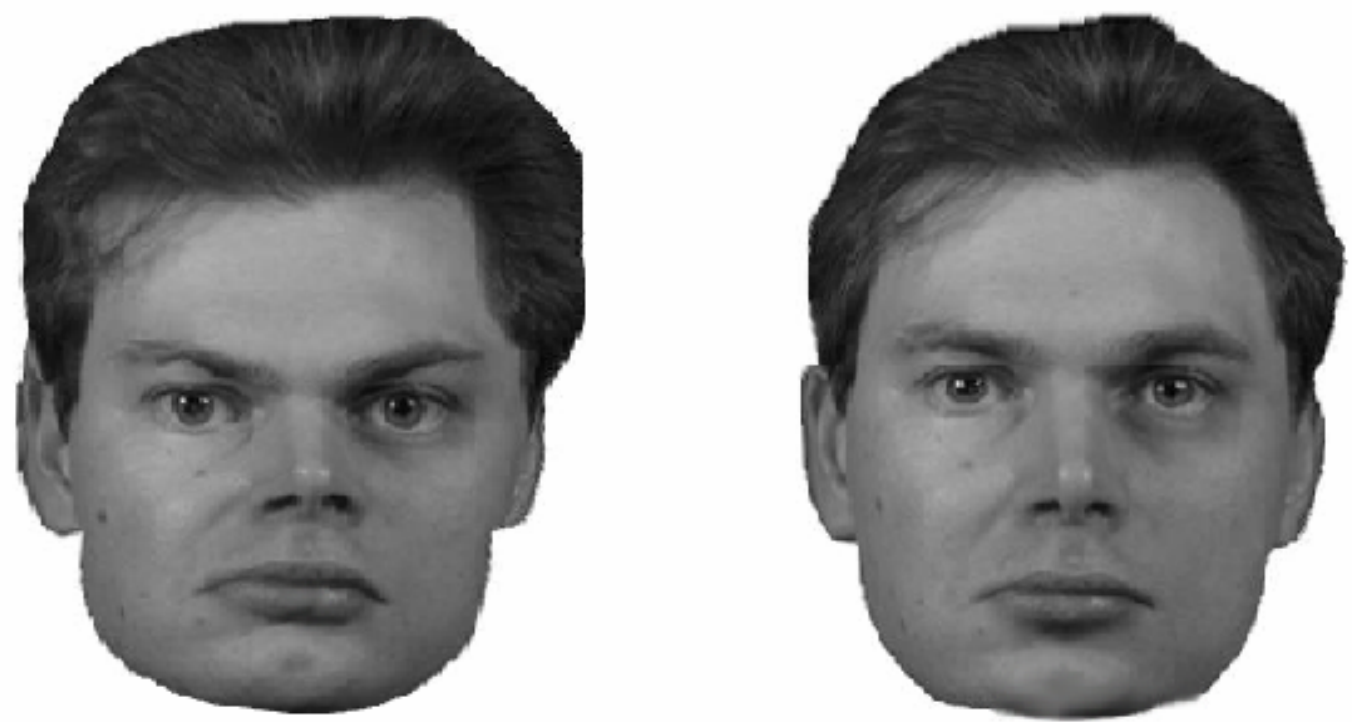

Figure 3. An example of two different faces changed along the caricature continuum (3a) and the oblique continuum (3b). The change shown is the same as a $100 \%$ change seen by the participants in this experiment.

Note that the Oblique and Caricature versions of a face will not in general be the same distance from the original: as drawn, the Oblique version of face $\mathrm{A}$ is closer than the Caricature version. However, because the faces are generated in pairs, the caricature version of face B will be the same distance from face B as the Oblique version of face A is from face A. Thus on average, the Oblique and Caricatured versions are the same distance from their original faces. We are concerned with determining which change is more detectable.

Procedure. Participants were tested individually. Each participant was tested on one of the 22 face sets containing 51 faces on the Oblique continuum and 51 faces along the Caricature continuum. Both continua covered the same physical distance but were centred on different identities. 
The faces were presented on the monitor in a pyramid formation with the target face presented at the top and the distracter and a copy of the target presented beside each other at the bottom. Which side the distracter and the copy of the target appeared on was randomised. All faces were presented on a white background with the top image scaled to a width of $6 \mathrm{~cm}$ and the two bottom images scaled to a width of $8 \mathrm{~cm}$.

Participants were instructed that they had to decide which of the bottom two faces was the same as the face at the top. Participants made their responses using the mouse to click on either a button marked 'left' or a button marked' right'. If the participant did not respond within 5 seconds then the program recorded the participant as not knowing the answer, as opposed to having answered wrongly.

On each trial the staircase program (created using the Bayesian adaptive psychometric methods toolbox; see Tanner, Hill, Rasmussen \& Wichmann, 2005) selected two face images from one of the two continua of faces. Trials containing faces on the two continua were intermixed although the program handled them using separate staircases. Faces were selected so as to traverse the veridical target (continuum midpoint) with one face selected from each side. The program then arbitrarily assigned one of the two faces as the target face and the other as the distracter. The program stopped after running 100 Oblique and 100 Caricature trials and the experiment data were recorded.

Design. The experiment was a within subjects design. The dependent variable was the percentage change from the veridical image that was just perceivable to the participant. The independent variable was the continuum type: in the direction caricature; or oblique to the direction of caricature.

Results

Participants required a mean of $68 \%$ change to reliably identify which of two stimuli was the same as the target. The mean percentage change required in the Oblique condition was $62 \%$ vs. $73 \%$ for Caricature. A paired t-test revealed that the difference was statistically significant, $t(21)=2.93, p<.01$.

\section{Discussion}

The results from Experiment 1 show that participants were more sensitive to changes made to a face in a direction oblique to the caricature vector. The just noticeable difference in the Caricature direction condition was $73 \%$ compared with $62 \%$ in the Oblique condition. This result suggests that information pertaining to the angular difference between two faces is important in discriminating between them. Thus supporting the notion that vector direction is a component of a face's identity and consequently the similarity of two faces is not just a product of their vector similarity based on vector subtraction.

Given that the experiment was counterbalanced to remove any effects of distinctiveness or of any particular stimuli there do not appear to be any obvious reasons to doubt the validity of these results. The key difference between this study and previously reported studies in this area (e.g. Carey, 1992; Lewis \& Johnston, 1998; Rhodes et al, 1998) is that participants were not required to say which face looked more like a known celebrity or make a judgement about similarity from memory; they were simply required to detect a difference from the target. 


\section{Experiment 2}

Experiment 2 set out to test whether the effect found in the first experiment could be replicated. In particular we wondered if using familiar faces would affect the result. The design of Experiment 2 was identical to Experiment 1 except that the stimuli were all famous faces. As we were unable to control this face set so carefully it was also interesting to see if the effect was robust enough to appear even when lighting and pose were not tightly constrained. Recognition rate and familiarity ratings for stimuli used in this study had been assessed previously with all the faces being recognised more than $80 \%$ of the time.

Method

Participants. The participants were another sample of 22 undergraduates from Cardiff University, School of psychology who signed up for the experiment as a partial fulfilment of course requirements. Participants were aged between 18 and 23 years, and all reported that they had normal or corrected to normal vision.

Materials. The basic experimental apparatus were the same as those used in Experiment 1. The experimental stimuli were constructed from 22 photographs of well known male celebrities selected from images found on the internet. The stimuli were selected so that they were all full frontal view photographs with relatively even lighting and high resolution. The set of faces were selected so as to have a similar age range as the unfamiliar faces used in Experiment 1. The Caricature and Oblique continua were constructed in the same way as was described for Experiment 1. Experiment 1.

Design and Procedure. Both the design and the procedure were identical to those in

Results

Participants required a mean of $49 \%$ change to reliably identify which of two stimuli was the same as the target. The mean percentage change required in the Oblique condition was $45 \%$ vs. $54 \%$ in the Caricature-direction condition. A paired t-test revealed that the difference was statistically significant, $t(21)=2.11, p<.05$.

Combined analysis

The results of experiment 1 and 2 were also subject to a Mixed model ANOVA with experiment (e.g. unfamiliar vs. familiar faces) as the between subjects variable and face type (Caricature vs. Oblique) as the within subjects variable. The analysis revealed a significant overall effect of both face type $F(1,14)=12.248 \mathrm{MSE}=76.118, p<.05$ and familiarity $F(1$, $14)=15.202 \mathrm{MSE}=271.917, p<.05$. However, there was no interaction between face type and familiarity $F(1,14)=0.07 \mathrm{MSE}=0.436, p=>05$.

\section{Discussion}

The results of Experiment 2 were in line with the results found in experiment 1 . They suggest that equal changes in physical space are not equal within perceptual space. Specifically it seems that vector direction from the norm has an important role in face perception and deviations in this direction are more disruptive to identity than equivalent physical changes that maintain the direction of the vector. 
The fact that the effect was found even for the uncontrolled celebrity stimuli used in experiment 2 suggests that the effect is relatively robust. It is clear from the results of the second experiment that changes were more easily detected in the celebrity faces than in the unfamiliar faces in experiment 1 . An average of $49 \%$ change was required for a just noticeable difference in experiment 2 vs. 68\% in experiment 1 . The combined analysis revealed that this difference was significant.

There are several reasons why one might expect a greater sensitivity to change in familiar faces than in unfamiliar faces. First, it may simply be that participants attended to the internal features more when the faces were familiar (e.g. see Young et al., 1985). Given that the degree of natural variation in internal features is likely to be less than the degree of variation in external features, which includes the jaw and hair lines as well as hairstyle, it would be reasonable to think that internal features would provide better cues for completing a subtle perceptual task such as the one reported here.

A second related reason for the improvement in the participants performance in experiment 2 is that familiar faces may be processed in a more holistic manner, partly due to the increased focus on their internal features (see Osborne \& Stevenage, 2008). However, this second explanation was not directly supported by the results as there was no interaction between familiarity and face type.

\section{General Discussion}

The two experiments presented here suggest that the angle between two norm-face vectors provides perceptually relevant information for the discrimination of the two faces beyond that provided by similarity based on vector subtraction. This finding is consistent with the notion that there is something special about the direction in which a face differs from the norm.

While the importance of vector direction has been implicit in accounts of face space, this is the first time that direct evidence for it has been demonstrated. Indeed, two previously reported studies to have investigated this question did not find such an effect (Rhodes at al., 1998; Lewis \& Johnston, 1998). As was discussed in the introduction, in these previous memory-based studies the number and proximity of exemplars other than the target face may affect a probe face's rated similarity to a known target. The present research attempted to avoid the question of exemplar density as participants were not required to say which face looked most like a known person or a previously seen person; rather they were asked to say which face was the same as a target that was present on the screen. It was hoped that this would make the task less susceptible to the problems of face recognition memory.

Indeed, although the stimuli used in the two reported experiments were not strictly lateral caricatures, these results speak directly to the previous research in this area. There is no reason to believe that there is any particular psychological significance given to the perpendicular or lateral directions, nor for that matter the anti caricature or caricature directions. The present research is simply interested in discovering whether there is a role for angle in differentiating two face vectors.

Although the role of vector angle has been included in a number of descriptions of a norm-based space (e.g. Rhodes, 1995; Rhodes et al 1998) it is not actually an obvious result of such a coding system (Craw, 1995; also see Valentine, 1999). As was discussed, the 
similarity between two vectors in a norm-based space is identical to the similarity between two points in an exemplar-space. Nevertheless the present findings are consistent with a model in which the similarity of two faces is provided by the pattern of activation across a range of encoded dimensions rather than a sum of their absolute similarity. This would seem to suggest that a whole face may not be treated as simply the sum of its parts but that there is an interaction between the components.

The current research opens up a number of further directions for investigation. For example, it is not clear if the dissimilarity due to a change in direction would be constant across the space or whether it would depend on the direction of the change. It is also worth considering whether the findings relate to holistic processing. Indeed, it has been suggested that holistic processing is affected by inversion (e.g. Young, Hellawell \& Hay, 1987) and it might be fruitful to investigate whether the observed effect interacts with orientation.

In conclusion, two experiments indicated that changes along the vector between the norm and a target face are more difficult to perceive than changes oblique to this direction. Given the apparent importance of the norm in such a model, this finding would seem to further support the evidence for the role of norm-based coding in face recognition. Importantly these findings provide the first direct support for a specific type of norm-based model; one in which vector direction is important beyond simple vector subtraction.

\section{References}

Benson, P.J., \& Perrett, D.I. (1994). Visual processing of facial distinctiveness. Perception. 23, 75-93.

Carey, S. (1992). Becoming a face expert. Philosophical Transactions of the Royal Society of London, Series B. 335, 95-103.

Craw, I.G. (1995). A manifold model of face and object recognition. In T.R. Valentine (ed.) Cognitive and Computational Aspects of Face Recognition, 183-203, London: Routledge.

Leopold, D.A., O’Toole, A.J., Vetter, T., and Blanz, V. (2001). Prototype-referenced shape encoding revealed by high-level aftereffects. Nature, 4, 89-91.

Lewis, M.B., \& Johnston, R.A. (1998). Understanding caricatures of faces. Quarterly Journal of Experimental Psychology. 50, 321-346.

Lewis, M.B., \& Johnston, R.A. (1999). A unified account of the effect of caricaturing faces. Visual Cognition, 6, 1-42.

McKone, E.M., Aitkin, A., \& Edwards, M. (1995). Categorical and coordinate relations in faces, or Fechner's law and face space instead? Journal of Experimental Psychology: Human Perception and Performance. 31, 1181-1198.

Osborne, C.D., Stevenage, S.V. (2008). Internal feature saliency as a marker of familiarity and configural processing. Visual Cognition. 16, 23-43.

Rhodes, G. (1995). Face recognition and configural coding. In: T. Valentine (Ed.) Cognitive and Computational Aspects of Face Recognition: Explorations in Face Space. pp. 47-68, London: Routledge. 

Press.

Rhodes, G. (1996). Superportraits: Caricatures and Recognition. Hove: Psychology

Rhodes, G., Brennan, S., \& Carey, S. (1987). Identification and ratings of caricatures: Implications for mental representations of faces. Cognitive Psychology. 19, 473-497.

Rhodes, G., Carey, S., Byatt, G., \& Proffitt, F. (1998). Coding spatial variations in faces and simple shapes: A test of two models. Vision Research. 38, 2307-2321.

Rhodes, G., \& Jeffery, L. (2006). Adaptive norm-based coding of facial identity. Vision Research. 46, 2977-2987.

Rhodes, G. \& McLean, I.G. (1990) Distinctiveness and expertise effects with homogeneous stimuli: Towards a model of configural coding. Perception. 19, 773-794.

Robbins, R., McKone, E., \& Edwards, M. (2007). Aftereffects for face attributes with different natural variability: Adapter position effects and neural models. Journal of Experimental Psychology: Human Perception and Performance. 33, 570-592.

Stevenage, S.V. (1997). Face facts: Theories and findings. The Psychologist. 10, 163168.

Tanaka, J.W., \& Farah, M.J. (2003). The holistic representation of faces. In M.A. Peterson \& G. Rhodes (Ed.), Perception of Faces Objects and Scenes (pp. 53 - 74). Oxford University Press.

Tanner, T.G., Hill, N.J., Rasmussen, C. E., Wichmann, F.A., Efficient adaptive sampling of the psychometric function by maximizing information gain, Proceedings of the 8th Tübinger Perception Conference, 106. (Eds.) Bülthoff, H. H., H. A. Mallot, R. Ulrich and F. A. Wichmann, Knirsch Verlag, Kirchentellisfurt (2005)

Tiddeman, B., Burt, D.M., \& Perrett, D. (2001). Prototyping and Transforming Facial Textures for Perception Research, IEEE Computers Graphics and Applications. 21, 42-50

Valentine, T. (1991). A unified account of the effects of distinctiveness, inversion and race in face recognition. Quarterly Journal of Experimental Psychology, 43A, 161-204.

Valentine, T., \& Endo, M. (1992). Towards an exemplar model of face processing: The effects of race and distinctiveness. Quarterly Journal of Experimental Psychology. 44A, 671-703.

Young, A.W., Hay, D.C., McWeeny, K.H., \& Flude, BV.M. (1985). Matching familiar and unfamiliar faces on internal and external features. Perception. 14, 737-746.

Young, A.W., Hellawell, D., \& Hay, D.C. (1987). Configurational information in facial perception. Perception, 16, 747-759. 